\title{
La naturaleza como obstáculo: perspectivas críticas del avance de la vivienda social e infraestructura vial sobre los humedales en la ciudad de Valdivia, Chile
}

\section{Nature as an obstacle: critical perspectives of the advancement of social housing and road infrastructure over wetlands in the city of Valdivia, Chile}

\author{
Rodrigo Hidalgo $^{1}$, Laura Rodríguez Negrete ${ }^{2}$, Alex Paulsen-Espinoza ${ }^{3}$, Voltaire Alvarado ${ }^{3,1}$
}

\begin{abstract}
Resumen
Discutir bajo los conceptos de un modelo científico reporta ciertas dificultades, sobre todo en el desarrollo de las reflexiones y las formas de mirar el espacio geográfico. El siguiente caso presenta el progreso en la expansión urbana de la ciudad de Valdivia, Chile, en el marco de las ofertas de ocupación excepcional en determinados espacios, de relevante valor natural, pero cuya aptitud comercial los hace aún más atractivas para las operaciones habitacionales y de infraestructura. Se observa, a partir de la evidencia recogida en la zona, que las articulaciones en la reproducción del capital en el espacio superan la barrera de la naturaleza, convirtiéndola permanentemente en espacios antropizados y convenientemente alterados, tornándola en una segunda naturaleza. Esta nueva versión del entorno abandona sus cualidades como obstáculo, resultando de ella zonas excepcionales para su análisis, como es el sector Guacamayo en la ciudad de Valdivia. La bandurria es la excusa para poner a prueba el marco conceptual que la ecología política ofrece para conocer del cruce entre sociedad y naturaleza.
\end{abstract}

Palabras clave: ecología política, segunda naturaleza, Valdivia, vivienda.

\begin{abstract}
Discussing under the concepts of a scientific model reports certain difficulties, especially in the development of reflections and ways of thinking the geographical space. The following case presents the progress in the urban expansion in the city of Valdivia, Chile, within the framework to offers an exceptional occupation in certain spaces with relevant natural value, but whose commercial aptitude makes them even more tasty for housing operations and infrastructure. It is observed, from the evidence gathered in the area, that the articulations in the reproduction of capital in space break natural barriers, converting it permanently into anthropized and suitably altered spaces, turning it into a second nature. This new version of the environment abandons its qualities as an obstacle, resulting in exceptional areas for analysis, such as the Guacamayo sector at the city of Valdivia. The bandurria is the excuse to test the conceptual framework that political ecology offers to know about the cross between society and nature.
\end{abstract}

Keywords: housing, political ecology, second nature, Valdivia.

Recibido el 31 de enero de 2018, aceptado el 22 de octubre de 2018.

Cómo citar: Hidalgo, R., Rodríguez Negrete, L., Paulsen-Espinoza, A. y Alvarado, V. (2018). La naturaleza como obstáculo: perspectivas críticas del avance de la vivienda social e infraestructura vial sobre los humedales en la ciudad de Valdivia, Chile. Investigaciones Geográficas, 56, 27-44. https:// doi.org/10.5354/0719-5370.2018.48409

1 Instituto de Geografía, Pontificia Universidad Católica de Chile. Av. Vicuña Mackenna 4860, Macul, Santiago de Chile.

2 Instituto de Arquitectura y Urbanismo, Universidad Austral de Chile. Campus Isla Teja, Valdivia, Chile.

3 Escuela de Geografía, Universidad Academia de Humanismo Cristiano. Condell 343, Providencia, Santiago de Chile. E-mail: valvaradop@docentes.academia.cl 


\section{Introducción}

El inicio de la primavera de 2017 es recibido por un corte general del servicio de energía eléctrica en un amplio sector de Isla Teja, ciudad de Valdivia, que se extendió por algunas horas. Junto a las molestias por el desperfecto, emergieron las vocerías de la empresa de distribución eléctrica SAESA -de propiedad privada, como la mayoría de los sectores estratégicos para la economía chilena- informando que lo acontecido tuvo su origen en la colisión bandurria contra un reconectador del tendido eléctrico que alimenta a Isla Teja ${ }^{1}$. Al revisar la línea de tiempo de la compañía SAESA en la red social Twitter se advierte una periodicidad de eventos similares bastante llamativa ${ }^{2}$.

Lo llamativo es el motivo de la interrupción, pues un pájaro acusa la fragilidad de las instalaciones ante estos episodios, lo que no deja ser preocupante para un sector estratégico como es la electricidad. Esto evidencia la capacidad dinámica de la naturaleza para imponerse en los procesos e hitos sociales que instalan lo urbano como condición dominante de ocupación territorial en zonas de diverso interés.

Más allá de las comunicaciones inmediatas de las redes sociales y el componente anecdótico que se presenta más arriba, se abre la posibilidad a intervenir en la problemática que enfrenta a la sociedad contemporánea con la naturaleza. Si las acciones ejecutadas por individuos e instituciones apuntan al control de aquello que se entiende como naturaleza, termina esta por constituirse como un objeto moldeable al servicio de la ocupación humana, muchas veces sustraída de su eje relacional y situado en la capacidad epistemológica de la Ecología Política, en el análisis de la relación sociedad-naturaleza.
El sector Guacamayo, emplazado hacia el sur oriente de la ciudad de Valdivia exhibe la tensión entre lo social y lo natural. El incidente de la bandurria en Isla Teja abre la metáfora para trazar rutas analíticas y comprensivas respecto a los objetivos y alcances de la Ecología Política como forma de conocimiento. Por lo tanto, este trabajo dialoga con determinadas propuestas teóricas y experiencias de caso enfocadas en la ocupación de entornos naturales por el desarrollo habitacional, contenido en el inventario de las viviendas sociales (sin deuda) y subsidiadas (con deuda hipotecaria) construidas en la zona y la infraestructura necesaria para asegurar movilidad, motilidad y conectividad, entre otros aspectos de lo cotidiano.

El obstáculo se configura en los humedales urbanos y periurbanos de Valdivia. Su caracterización como áreas de conservación y protección silvestre colisionan con los objetivos de expansión habitacional, al mismo tiempo que reflejan un valor de paisaje que tensiona sus usos de producción material con los de construcción subjetiva. La naturaleza sería, por lo tanto, un objeto más del desenvolvimiento urbano; un hito para posicionar diversos tipos de vivienda y jugar con la producción/construcción de valor en un entorno que se lee y presenta como natural. Tal como lo son las bandurrias.

\section{Materiales y métodos}

Los pasos metodológicos llevados a cabo para el análisis del problema aquí planteado se han constituido de la siguiente manera. En primer lugar, se analizaron todos los proyectos de inversión en infraestructura entre el 2007 y el 2017 en el área de estudio. De esta manera, se confeccionó una base de datos con la siguiente información: año,

4 Ave de gran tamaño, corresponde a un Ibis y se les conoce como ave zancuda por el largo de su pico.

5 La cuenta de Twitter de SAESA (@Saesa_) indica al menos seis episodios similares entre los meses de noviembre a diciembre de 2017: 8, 17, 23 y 26 de noviembre; 18 y 29 de diciembre de 2017. El episodio de la bandurria corresponde al primero de ellos. 
nombre de proyecto, clasificación de la inversión, inversión total, fecha de inicio de obras, fecha términos de obra y nombre del contratista.

Los datos aportados por esta base de datos permitieron identificar en primer lugar la evolución de los proyectos de inversión; en segundo lugar, los tipos de proyectos de inversión en infraestructura (mejoramiento; construcción; reposición; conservación); posteriormente, establecer una relación entre el espacio intervenido y los tipos de obras realizadas; y finalmente, identificar los actores que participaban en dichos proyectos.

En una segunda etapa del proceso metodológico, se elaboró una base de datos con los conjuntos de vivienda sociales entregadas entre el 2000 y el 2016 en el área de estudio. Esta se construyó a partir de los datos aportados por la Municipalidad de Valdivia, específicamente por la Dirección de obras de dicha entidad, a través del Portal de Transparencia. La base de datos estaba compuesta por los siguientes campos: nombre conjunto de vivienda social, dirección, tipo de vivienda, $\mathrm{M}^{2}$ total, $\mathrm{M}^{2}$ por unidad, inversión, número de viviendas, constructora y año.

Luego de la construcción de esta base de datos, se llevó a cabo el proceso de geocodificación de los conjuntos de viviendas sociales. La geocodificación es un proceso geomático que se realiza a través de la herramienta ArcGIS 10.5. Este proceso es la transformación de una descripción de una ubicación en un punto en la superficie terrestre. Considerando la dirección de la base de datos, esta herramienta geomática indica un punto en una matriz tributada de direcciones en el software ArcGIS 10.5. Esta matriz tributada es un shape ${ }^{4}$ con los ejes de calles, en donde la base de datos ingresada busca la dirección para generar una expresión espacial por medio de un punto u otro elemento en la cartografía.

Esta geocodificación permitió identificar los lugares en donde la presión inmobiliaria estaba generando nuevos nichos de renta. Considerando la trayectoria espacial de esta inversión, se establecieron posibles sectores en donde la presión existente sobre las condiciones de sitio en Valdivia. Además de ello, se cruzaron coyunturas históricas con los datos de las viviendas e infraestructura para identificar los momentos en donde la acumulación ya no era posible, dando paso a procesos de sobreacumulación en nuevos nichos de rentas.

Finalmente, se analizaron diversos informes, tanto de agentes privados como públicos, para delinear la racionalidad de las intervenciones por medio de la vivienda e infraestructura en el área de estudio. Los reportes analizados fueron los entregados por la Dirección General de Concesiones del Ministerio de Obras Públicas; y ATISBA Estudios y Proyectos Urbanos que, en su cuarta sección, se encarga de Guacamayo como sector (2005). Estos informes aportaron insumos necesarios para identificar los objetivos de los proyectos, así como también las características de la construcción de infraestructura y vivienda en el área de estudio. Con el análisis de estos documentos se perseguía establecer las conexiones entre los agentes públicos y la consecuente inclusión de aquello que la literatura ha denominado actores no autorizados en la gestación y puesta en marcha de las políticas públicas (Zunino, 2006; Swyngedouw, 2011a; Silva, 2010).

La Geografía y la politización de la naturaleza. Por sí sola, la naturaleza ejerce el poder a través de sus dinámicas, procesos y productos. No existe un estado más puro de energía que el natural, siendo sus leyes un sólido testimonio. En Dialéctica de la Naturaleza, Friedrich Engels (2017)

4 Nombre de la extensión de los archivos que genera ArcGIS 10.5.

5 Corresponde a una solicitud de Transparencia Activa de los autores para el periodo entre 2000 y 2017. 
desarrollan algunas teorías y leyes para explicar los flujos de energía que transforman la materia, cuyo permanente movimiento posibilita la creación y destrucción de la vida, por ejemplo. Este aporte, entre otros, considera también el evolucionismo más reflexivo respecto a la técnica y la transformación de la naturaleza, no sólo al servicio de la producción y acumulación de capital, sino también en el marco de la comprensión racional de las formas de ocupación de la superficie de la Tierra, su relación con el Universo, el orden y caos como principios antagónicos pero sinérgicos, entre otras características (Engels, 2017).

La racionalidad evidente tras estas leyes abre la puerta a la politización de la natural, pues convierten sus procesos y dinámicas en esquemas de base para la administración territorial del poder. Bajo la lógica de Engels, son estas leyes las que sustentan las capacidades de control social sobre lo natural, lo que en trabajos de Reclus y Vidal de la Blache aparece como espacio geográfico, al estar la naturaleza intervenida por la razón y convertida en objetos de un sistema social superior a su propia esencia (Reclus, 1875; Vidal de la Blache, 1902; Correia de Andrade, 1985). El discurso geográfico establece, también, sus formas para el debate del mundo que está observando en movimiento para la segunda mitad del siglo XIX.

A estos último se suma la relación biologicista y determinista decimonónicas, que detallan los flujos de energía y materia vinculados al territorio como manifestación espacial del poder. Esta idea, conocida como "teoría dérmica sobre las fronteras del Estado" (Delaney, 2005, pp. 34-36), supone la apropiación de lo natural como una condición inevitable del desarrollo y progreso humanos, tal como lo plantea Ratzel en su ensayo sobre las leyes de crecimiento de los Estados, base teórica que luego será discutida por Mackinder (1987), Raffestin (2015) y Santos (2000).
El poder no se desvaneció del debate geográfico durante el siglo siguiente, más bien dio un salto en la escala local de estos ejercicios del poder, colocando a la posibilidad geográfica como esencia en su tránsito espacial (Kay \& Kenney-Lazar, 2017).

La objetivación de la naturaleza no es, por lo tanto, un argumento edificado en los últimos 40 años. Por el contrario, el discurso geográfico más radical inició su rescate en la década de 1970 desde las definiciones de Reclus contenidas a lo largo de su obra y, como lo plantea John Clark, el anarquista francés inventó la ecología social sin saberlo (Clark, 2015). Uno de los trabajos clave en esta construcción es el de Murray Bookchin (1978) donde se discute el sentido crítico de la ecología como aparato del poder y sus vinculaciones con el pensamiento libertario de finales del siglo XIX, al mismo tiempo que se reafirma la posición de la ecología como un ejercicio científico de equilibrios imposibles de ser practicados en contextos gobernados por la técnica industrial (Bookchin, 1978, p. 28).

La politización de la naturaleza se sitúa en esta discusión, al convertirse en un factor del poder. A partir de la producción/ construcción/ individual o colectiva del valor sobre determinados objetos situados en determinados espacios, se organiza una armonía artificial en ellos que, en relación con su conjunto o singularidad, genera tensiones y disputas entre agrupaciones sociales interesadas (Coombes, Johnson, \& Howitt, 2012). De ello emerge otra naturaleza, ahora transformada y convertida en un objeto sin esencias y desequilibrada en sus leyes, un objeto capaz de reproducir el capital (Postone, 2007). Este sería el objeto de estudio de la Ecología Política: el conflicto sobre lo natural devenido de la rugosidad social, desatado por el control y ejercicio del poder en una determinada situación espacial (Sultana, 2011; Demaria $\&$ Schindler, 2016). Es aquí donde empieza el problema. 
La naturaleza: referencias $y$ posicionamientos desde la Ecología Política. Una crítica de base para la Ecología Política está en la relación escalar que tiene con sus casos de estudio, que hace de sus lecturas una descripción con tendencia a la ubicuidad. Esta crítica tiene un fundamento en la escala relacional de los estudios desde la Geografía más radical, que examina precisamente las vinculaciones y transacciones entre el espacio y sus condiciones de sitio, donde lo social y lo natural están entre sí producidos y alterados. Posicionamiento que no está en las recientes oleadas alentadas por los trabajos de Swyngedouw (2011b), Heynen, Perkins, \& Roy, 2006), Kaika \& Ruggiero (2015) o Castree (2007). Por el contrario, se localizan en el radar de las naturalezas desarrolladas tempranamente por Reclus (Clark, 2015), que tienen su base en la idea de Baruch Spinoza sobre una naturaleza socializada como sistema armónico gobernado por leyes (Spinoza, 1675/2011, pp. 45-46). Estos actores de cambio, que por sus respuestas contrahegemónicas responden a quienes dominan la naturaleza, son el objeto de estudio de base en la Ecología Política. Las comunidades ancestrales ocupan un rol fundamental para estos fines, pues son quienes se enfrentan a los agentes del capital corporativo, cara a cara y en su propio territorio (Budds, 2004; 2012; Camacho, 2012; Prieto, 2015; Wilson \& Bayón, 2017). Otro tanto acontece con los estudios de Ecología Política Urbana, donde el derecho a la ciudad y la garantía en los accesos a recursos logra caracterizar las tramas del poder y de la invención de una nueva naturaleza, inédita por su capacidad metabólica y resiliencia, como es el caso de las ciudades del Norte y Sur Global (Lawhon, Ernstson, \& Silver, 2014)

El desarrollo epistémico de la Ecología Política está situado, en parte, sobre el fundamento antropológico de cultura y liminalidad de la etnografía, que se ocupa del sujeto de estudio como objeto de su propio contexto. Esto es visible en los trabajos de antropología urbana, donde estas representaciones están calibradas por modelos explicativos propios de la Geografía Urbana (Signorelli, 1999). Bajo estos supuestos es que se propone reconocer el piso geográfico en los discursos de la Ecología Política, particularmente sobre la reducción de escala relacional y la interacción de los agentes productores de espacio, que es la problemática que aborda este trabajo.

El sitio, su condición y la propuesta crítica. El ejercicio sobre estas cuestiones responde a la vorágine interdisciplinaria de quienes la trabajan y la estructura de defensa en toda revolución científica contemporánea (Lakatos, 1998). La Geografía, como ciencia del conflicto, aborda la relación entre sociedad y naturaleza como estructura clave en la definición del espacio geográfico, particularmente en el estudio de la ocupación humana de la superficie terrestre, que no puede ser armónica ni ubicua (Dollfus, 1976). El espacio es contradictorio porque cada área o polígono es único e irrepetible.

Uno de los aportes clave en la actualidad sobre esta condición de sitio está en la ecología del paisaje, situada en procesos socioecológicos pero trabada en relaciones geográficas de poder complejas y de base en el modelo neoliberal que, de cierta forma, han delineado la investigación respecto a los cuerpos de agua capturados por la expansiva persecución urbana, que en rigor es lo que acontece. Los humedales, por ejemplo, permiten caracterizar las tensiones entre lo social y lo natural.

El caso de Valdivia es clave para interpretarlo, pues las memorias de la década de 1920 sobre la ocupación de poblaciones esporádicas en su entorno $\mathrm{o}$ rellenándolos para transformarlo en favor de la ocupación, señalan a los pantanos como suelos de pésima calidad, arrojados al abandono y contrarios a toda forma higiénica que estuviera en boga para la época (Hidalgo, 
Rodríguez, \& Alvarado, 2018). Cincuenta años más tarde, los planes de reconstrucción en desarrollo con posterioridad al terremoto de 1960 señalaron a los humedales como espacios frágiles para el emplazamiento de conjuntos habitacionales que, sumado a procesos de basculamiento litoral en el frente fluvial, reestructuraron las lógicas de ocupación que anteriormente habían tenido estas poblaciones (Espinoza \& Zumelzu Scheel, 2016).

Los humedales no serán zonas prioritarias de conservación silvestre, sino hasta bien avanzado el último cuarto del siglo $\mathrm{XX}$. Bajo una lógica de bienes comunes, caracterizan la crítica pertinente que, desde la Ecología Política se ha trabajado en el análisis de los bienes comunes y el sentido de la objetivación de la naturaleza (Walker, 2005; Turner, 2017), que no es una situación teórica reciente (Postone, 2007). En rigor, el énfasis ha estado en aquellos trabajos que han estructurado una racionalidad territorial sobre los humedales, discutiendo su condición de objeto y reconociéndolos como órganos clave en los sistemas ecológicos (Rojas, Sepúlveda-Zúñiga, Barbosa, Rojas, \& Martínez, 2015). Entonces, si bien operan como bienes comunes desde su forma como órgano natural a proteger, su mayor tensión está cuando se convierte en cosa desde la cual se genera o extrae valor.

Estos puentes entre Geografía y Ecología Política son frágiles y por ello se plantea al inicio de esta sección una recuperación teórica de las bases geográficas de la ecología social. Desde ahí se advierte el papel de la Ecología Política contemporánea, sea urbana o no, de estar en la discusión más reducida en escala, lo que aportaría precisamente a la condición de sitio, que se define como la capacidad del medio natural para acoger las actividades humanas, la desagregación en capas de hidrología, geomorfología y biomas presentes, configurando la fusión en el propio sistema natural, intervenido y reproducido por la presencia de la actividad humana (Dollfus, 1976). Se entiende que los aportes de la Ecología Política permiten acceder al plano de la denuncia y crítica; mientras que por el curso de la Geografía concurren los elementos relacionales del espacio geográfico, que acusan los desequilibrios propios de las dimensiones físicas y humanas en su producción o construcción.

El problema es la escala, ya que mientras la Geografía se instala desde el sitio en la organización de las jerarquías, en la base de la Ecología Política el sitio es replicable y comparable, desatendiendo el palimpsesto que da sentido de lugar y territorio al espacio geográfico. No pasa por concentrar más o menos referencias bibliográficas sobre el tema, sino por la verificación de las metodologías que responden a condiciones de sitio caracterizadas elementos que tienden a repetirse: un recurso natural en disputa, donde los antagonistas están representados por una empresa transnacional y una comunidad con arraigo espacial, generalmente definido por sus usos ancestrales y relaciones de propiedad (Bolados \& Babidge, 2017; Romero, Videla, \& Gutiérrez, 2017; Schorr, 2018).

Entonces, el ejercicio descriptivo sobre el sector Guacamayo de Valdivia, sus humedales y desarrollo de infraestructura en torno a la densificación habitacional, propone discutir con estas categorías, las de condición de sitio, ubicuidad y poder, los discursos geográficos y sus encuentros con la Ecología Política. Lejos de una polémica, se busca alimentar el debate desde un caso de estudio fuera de las bases tendenciales contemporáneas.

\section{Las bandurrias como metáfora del poder} en el espacio. Tanto la vivienda como la infraestructura vial representan objetos claves en el ejercicio del poder sobre lo natural. Como se ha planteado, en los trabajos de Ecología Política destacan la crítica a la expansión urbana inorgánica (Angelo \& Waschmuth, 2015), la distribución social del poder entre lo político, la política y la policía como ciclos del espíritu social en el espacio (Swyngedouw \& Heynen, 2003), y la discordia entre la ecología como medio y 
lo político como fin en la conceptualización del conflicto (Paulson, Gezon, \& Watts, 2003). Estos elementos que dan marco al sentido de la denuncia y crítica desde la Ecología Política representan, entre otros, distintas miradas para leer la tensión sociedad-naturaleza en el sector Guacamayo de Valdivia.

Más allá de la metáfora que involucraba a la bandurria, el problema de la naturaleza como obstáculo para el desarrollo se sitúa, por una parte, en la producción de naturaleza con fines rentistas (Smith, 2006). Esta lógica es la promotora de la inversión en obras de escala media que convierten pequeños entornos naturales, frágiles como los humedales, en zonas de alto valor por sus líneas de paisaje, como acontece en Guacamayo. Son entornos que terminan colonizados por viviendas subsidiadas que precisan de equipamiento para arrancar un proceso paulatino urbanización e integración a la ciudad, en este caso con Valdivia. El peso de lo político, como acto desde los agentes hacia el piso de la pirámide social, influye en la descomposición de lo natural, pues deja de serlo en su versión prístina convirtiéndose en una segunda naturaleza funcional (Hidalgo, Camus, Paulsen-Espinoza, Olea, \& Alvarado, 2016).

La presión del capital por expandirse hacia el mayor valor probable es inevitable. Si se revisan el movimiento de las viviendas sociales primero, desde 1980 hasta 2004, y subsidiadas después en Valdivia, posterior a 2004, se advertirá que la cubierta natural fue clausurada en tanto obstáculo para el progreso. No fue sino hasta los años 2000 y posteriores que el régimen de utilización de los suelos se movilizó hacia el sur y cono nororiente de la ciudad, sobre los humedales y contra toda lógica conservacionista o patrimonialistas sobre estos espacios.

Como se observa, y lejos de ser un obstáculo, el asiento residencial en Valdivia parece depender de dos factores. El primero está en que la apertura de suelos con fines habitacionales es una necesidad de inversión y modernización dirigida esencialmente desde la policía que, organizada al servicio de los dispositivos de poder que se cuajan en lo público, gestiona la naturaleza politizándola y convirtiéndola en polígonos con un valor agregado a lo que podrían ser y no a lo que son. Es la estrategia de control y poder en la ecología social que trabaja la Geografía: el posibilismo de un entorno frente a la técnica y la transformación para asegurar valor inmediato y plusvalor futuro.

Lo segundo emerge en el acceso a estos nichos de naturaleza, lo que en sí produce espacio urbano como tal. Las obras de infraestructura representan una cara del desarrollo que en Chile ha sido clave para el sostenimiento de las economías de escala regional, al servicio de un modelo rentista y extractivo. Al servicio de la inversión privada y del incentivo a la creación de mayor conectividad, el diseño de licitaciones privadas por parte del Ministerio de Obras Públicas chileno (MOP) representa el éxito estratégico del Estado neoliberal de los últimos 30 años.

Un dato clave de esta penetración material de obras públicas está en que entre 1992 y 2012 se adjudicaron 82 proyectos para desarrollar en la zona de Guacamayo, con una inversión público-privada de 19 mil millones de pesos. Subdividiendo esta bolsa en sectores, se destacan vialidad interurbana, autopistas urbanas e infraestructura para el transporte público, las que se llevan el $85 \%$ de todo el gasto asignado desde la agenda de la Dirección General de Concesiones del MOP para el periodo señalado (20002017). Esto sin considerar al naciente Fondo de Infraestructura que, desde 2018 en adelante, recibirá la finalización de las obras concesionadas para depositarlas nuevamente en el mercado de quienes deseen administrarlas y rentar de ellas.

Ambos casos manifiestan las redes de inversión que posibilitan el aseguramiento 
del valor que la naturaleza entrega por su objetivación. El poder no está definido por un mapa delimitador de fronteras, sino por los impulsos de una política más bien rentista que expresa su poder en la colonización de una naturaleza antes desechada y hoy altamente valorizada. Como lo plantea Swyngedouw (2014), el problema de las gobernanzas locales está en su cooptación por parte de niveles mayores de policía, en el sentido de los agentes que delinean las estrategias de transformación de lo natural y la clausura de lo prístino, siempre bajo la idea de expandir los espacios fértiles para la extracción-reproducción de renta inmobiliaria, como lo es en Guacamayo.

La policía en acción. Antes de entrar a la acción de la policía es necesario hacer una genealogía de su utilización. El concepto de policía no es nuevo. Su evolución ha tenido cambios considerables durante las últimas décadas y sus orígenes se remontan al siglo XVI. Las primeras actuaciones de este tipo de poder (dentro de muchas otras formas del poder) se dan en el contexto de las funciones del conservador y reformador general de la policía. De esta manera, las funciones que cumplían estos eran en un primer momento "ocuparse de la instrucción de los niños y los jóvenes, de los pobres, de la salud pública, de los accidentes, de las normas del comercio y de la fabricación, de los bienes inmobiliarios" (Castro, 2011, p. 314).

Según Delamare (como se citó en Castro, 2011) la policía se ocupaba inicialmente de los dominios religiosos, costumbres, salud y las subsistencias, las ciencias y las artes liberales, el comercio, las manufacturas y las artes mecánicas, los domésticos y los obreros manuales, el teatro y los juegos, y el cuidado de los pobres" (Castro, 2011, p. 314). No obstante, Foucault cuestionará y modificará la interpretación de la policía en sus actuaciones hacia el siglo XVII en adelante.

Si hacia el siglo XVII la racionalidad política coloca los ojos sobre lo urbano como medio para mercantilizar las relaciones sociales ¿qué ocurre a fines del siglo XX e inicios del XXI con respecto a las actuaciones de la policía en el entorno urbano y su relación con el capital?

Para tales efectos, es necesario precisar el siguiente contexto. Luego de la caída de los socialismos a inicios de la década de los noventa, se comienzan a generar procesos de cambio a nivel político en las formas de gestionar los Estados. De tal manera, es posible advertir un progresivo cambio de estados democráticos a entes más bien administrativos (Swyngedouw, 2014). Esta reordenación implica algunos cambios en la gobernanza neoliberal, es decir, fueron emergiendo modos consensuados de elaboración de políticas y nuevas configuraciones institucionales articuladas en lo público y privado.

En este contexto de reestructuración, los nexos entre el estado y la sociedad civil operan a distancia. Además, se originan nuevos arreglos de la gobernanza a nivel escalar e inter-escalar, comprometiendo espacios subnacionales, supranacionales y la existencia de una "gobernanza más allá del estado" (Filibi, 2005). Como ha sido señalado, la reinvención de la política en el orden social global ha sido penetrada por la gobernanza de los actores "no autorizados" tales como consultores, think tank, expertos y administradores, entre otros (Zunino, 2006). Los números en Valdivia dan cuentan del interés por ingresar al negocio de la naturaleza, desde el incremento de asesorías y consultorías dedicada a la infraestructura (figura 1).

La policía ha comenzado a incluir voces que no están legitimadas por la comunidad social, pero que si son útiles para incluirse en las estrategias y mecanismos de dominación de la naturaleza. Esto es parte de su espíritu. En tal sentido, la policía es un concepto que apunta a la organización de la "reunión de los hombres en comunidad y su consentimiento, y descansa en la distribución jerárquica de 


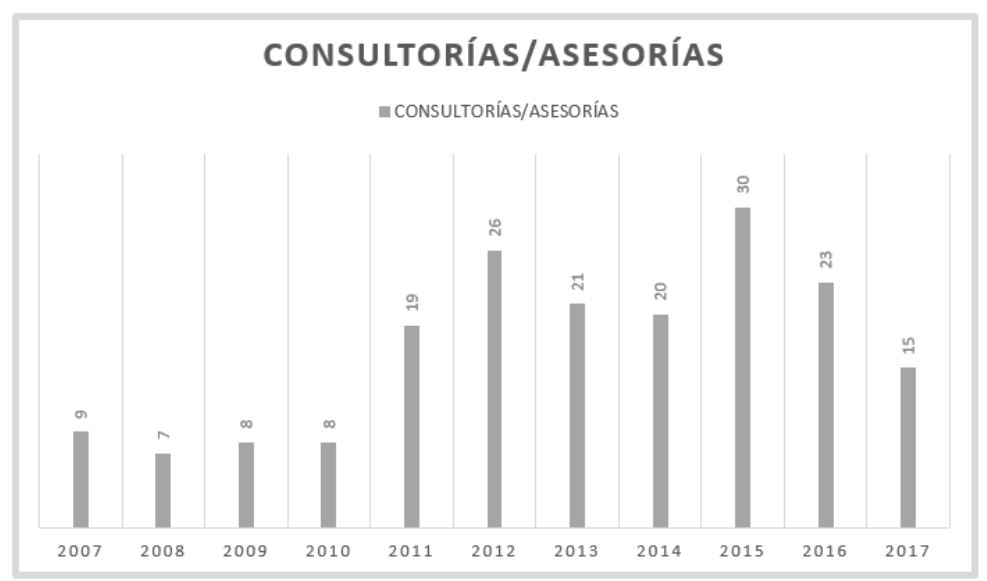

Figura 1. Asesorías y Consultorías en materia de infraestructura vial en Valdivia entre 2007-2017.

Fuente: Elaboración propia en base a Dirección de vialidad, Valdivia, MOP, 2018.

Figure 1. Consultancy and Advisory on Road Infrastructure in Valdivia between 2007-2017. Source: Own elaboration based on MOP, Valdivia, 2018.

lugares y funciones" (Rancière, 2006, p. 17). Este concepto no sólo se constituye en un mero acto de distribución, sino que responde a una multiplicidad de actos y procesos que se caracterizan por "conflictos y tensiones, nunca se encuentra totalmente cerrada y abraza no sólo la noción tradicional del Estado, funciones y actividades estatales, sino también la espontaneidad de las relaciones sociales" (Swyngedouw, 2011b).

La expansión de esta noción de Estado ha llevado a incluir a estos agentes no reconocidos, los cuales han sido parte importante de la elaboración de políticas públicas y la conformación de un paisaje que ofrece más nichos de rentas para los privados, que espacios para el "habitar", en el sentido tradicional de la naturaleza en Geografía, descrito al inicio.

En este contexto, el rol de los agentes al servicio de la policía y la gobernanza local se refleja en el perfil de la consultoría como pilar en la creación de información estratégica para el aprovechamiento de lo natural en tanto elemento distintivo de un entorno determinado. El trabajo de la consultora ATISBA en 2005 es un claro ejemplo de la expansión concertada hacia lo natural, donde todo obstáculo es superado por la capacidad inversora y ejecutora tanto del municipio, el MOP y los operadores habitacionales. ATISBA lo dibuja de la siguiente manera:

"[...] el sector analizado corresponde al Seccional Guacamayo, que comprende 624 hectáreas, ubicadas en el extremo Sur de la ciudad. El seccional, propone modificar el límite urbano vigente, extendiéndolo hasta los ríos Guacamayo y Angachilla. En este territorio se destinan 373 hectáreas para suelo urbanizable, reservando el resto para áreas verdes y protección de humedales. La cabida del seccional es de 10.659 viviendas, incluyendo unidades sociales, subsidios y viviendas privadas. Con esta propuesta, Guacamayo se transforma en la principal reserva de suelo de Valdivia y en el principal terreno para cubrir el déficit de la comuna". (ATISBA, 2005, p. 4).

No cabe duda de que el rol de los agentes privados está tras el espíritu efectivo de la gobernanza, otro de los conceptos clave en la ecología política. La relación espacial 
presente no es tan evidente como parece a simple vista, sino que encarna lógicas de efectividad y eficiencia en la gestión de la policía sobre el espacio urbano posible, el de los humedales en su función capturadora del valor de la conservación, donde rebota el privilegio de colindancia topológica entre lo residencial y lo natural (Roth \& Dressler, 2012). Se abandona entonces la primera naturaleza, esta deja de ser prístina y pasa convertirse en una segunda socializada, politizada y disputada.

Para 2005, el sector Guacamayo no registra números halagüeños en densidad de viviendas. Estudios como el de ATISBA declararon en su momento que la oferta podría aumentar en un $68 \%$ el total de residencias ofrecidas, donde las de carácter subsidiado y social disminuiría en favor de los "estratos sociales C2-C3 [que] subirían su participación de $28 \%$ a $51 \%$ [...] mientras que los estratos D y E disminuirían de $62 \%$ a 45\%" (ATISBA, 2005, p. 64).

Esta apuesta por un mayor impulso a sectores medios, sujetos de crédito y con capacidad de endeudamiento hipotecario refleja el valor que el humedal tiene en sí mismo como objeto asegurador del valor en la zona. La evidencia más contundente de esta situación la aporta el Ministerio de Vivienda y Urbanismo (MINVU) que, en 2016, declara a través de su ministra que Guacamayo representa "un barrio que es lo que quisiéramos tener todos los chilenos: una vivienda de calidad, un barrio donde las personas se conozcan y fortalezcan sus redes, en una ciudad donde efectivamente preste los servicios y conecte a los habitantes" (MINVU, 2016).

Por otra parte, la fragmentación del paisaje vista desde el aprovechamiento del humedal como herramienta de segmentación de los estratos socioeconómicos presentes en Guacamayo, permite ver la tendencia inequívoca señalada tempranamente por ATISBA y que se reitera sin grandes modificaciones en la actualidad. Los brazos del cuerpo de agua contienen grupos diferenciados de población, beneficiando en el acceso a las viviendas de mayor costo por su proximidad a las obras de infraestructura y confinando en áreas con una más alta densidad a los segmentos $\mathrm{C} 2$ y C3, tal como lo anunciara la consultora, cual oráculo en la producción urbana de la ciudad de Valdivia. Esto se aprecia en la siguiente figura.
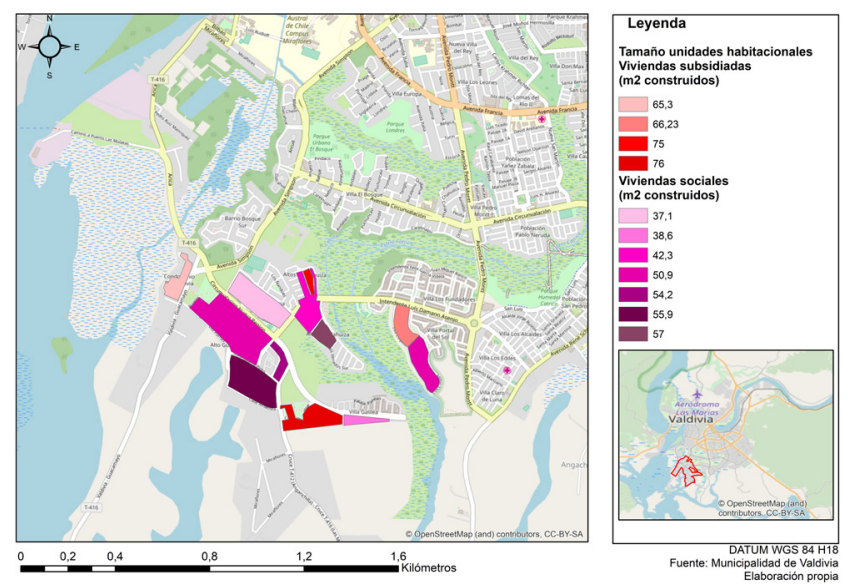

Figura 2. Conjuntos de viviendas por año de entrega sector Guacamayo, Valdivia. Fuente: Elaboración propia con base en datos Municipalidad de Valdivia, 2017.

Figure 2. Social Housing groups by year of installment Guacamayo, Valdivia. Source: Own elaboration based on Municipality of Valdivia data, 2017. 
La condición de obstáculo que se ha propuesto sería, entonces, relativa con relación a estas evidencias. Sin embargo, es pertinente sostenerla debido a que, si la naturaleza está politizada y mercantilizada de la forma en que sería vista desde la ecología política, también se debe destacar la aptitud que tiene como instrumento para otras fragmentaciones desde su función paisajística. Si la condición de sitio es una bisecada en cuanto a la presencia habitacional, la diferencia fragmentaria es el resultado de otros objetos urbanos. Aquí es donde las obras viales establecen los saltos de renta, evaluando las eficacias de la policía en el territorio de Valdivia.

Las fronteras de la naturaleza: vivienda e infraestructura vial como frente de tensión urbana.

Los informes como los de ATISBA fueron y son parte importante del desarrollo de nuevos proyectos urbanos de vivienda, infraestructura y equipamiento, entre otros. Pero también para la creación de sectores espaciales de acumulación, reproducción y sobreacumulación del capital en su alianza con la gobernanza neoliberal. Como se mencionaba más arriba, los proyectos de infraestructura representan un hito importante no solo para el desarrollo urbano, sino que también para el crecimiento económico del país.

Como señala Harvey (2012) los ciclos económicos y sus constantes crisis, precedidas de un crecimiento inmobiliario, se caracterizan por la circulación de capital fijo, trabajo y producción, las cuales a su vez necesitan de un sistema de créditos especulativo, consumo e interés (Harvey, 2012). Cuando la acumulación se hace insostenible y deviene en crisis, la urbanización se presenta como la solución para la absorción de los excedentes de capital. Esto ha sido parte de varios países de América Latina en materia de infraestructura. Luego de la crisis del 2008 el capital busca espacializar sus excedentes a través de infraestructura, aumentando los niveles de inversión.

Esto fue parte de la ideología neoliberal que se profundiza entre 1990 y el 2006, y que persiste en la clase política en Chile. No es menor que el expresidente de la república (2000-2006) Ricardo Lagos haya señalado durante su campaña presidencial del 2017 , que "es esencial recuperar los niveles de crecimiento económico y el ritmo de inversión en infraestructura que teníamos una década atrás" (Emol, 2017).

Valdivia se incorpora en este circuito del capital y, por lo tanto, es una frontera de la naturaleza que comienza a retroceder a raíz del avance del frente de guerra urbano que ejecuta la infraestructura vial promovida por los informes de los actores no reconocidos, como los denomina Zunino (2006). Luego de la crisis asiática de 1998 era necesario buscar nichos de renta para distribuir los excedentes de capital propios de los momentos de acumulación. Hacia el 2002 la zona de Guacamayo presentaba una baja ocupación y una densidad de carácter rural (ATISBA, 2005). La condición de sitio urbanizable era potencialmente buena para los proyectos que legitima la empresa consultora ATISBA hacia el 2005.

Esto da pie para el incremento de las inversiones en vialidad en la comuna de Valdivia. Entre el 2007 y el 2015 el incremento de las inversiones en vialidad comienza a ser considerable, como se aprecia en la tabla 1.

Las condiciones de sitio de Valdivia la han hecho un lugar en donde la infraestructura y las viviendas han comenzado un proceso de presión constante a los humedales. Como se aprecia en los anteriores mapas, viviendas sociales y económicas se han construido cerca del humedal Angachilla y Los Conquistadores. Para el caso del primer humedal, la edificación de viviendas sociales se produce entre el 2002 y el 2016; mientras que, para el caso de 
viviendas económicas, estas se construyen entre 1985 y 2006.

Ante tal panorama, es posible advertir que los procesos de edificación de viviendas y el aumento de inversión en vialidad se incrementan de la misma manera y con ello la presión sobre los humedales. No es menor que el conflicto por el tema de los humedales siga siendo denunciado por quienes habitan en Valdivia, sobre todo a partir de la infraestructura que se ha construido en los últimos años. Un grupo de vecinos ha alertado al gobierno local sobre las inundaciones que se han producido en la avenida Circunvalación Sur y que afectan a viviendas sociales aledañas (Jara, 2015).

La avenida Circunvalación Sur se proyecta luego de la identificación de nichos de renta en Valdivia (a partir del informe de ATISBA), comenzando sus estudios de factibilidad hacia el 2008 (tabla 2).

\section{Tabla 1}

Número de proyectos de inversión en vialidad en Valdivia (2000-2017). Fuente: elaboración propia en base a Dirección de vialidad, Valdivia, MOP, 2018.

Table 1

Number of road investment projects in Valdivia (2007-2017). Source: own elaboration based on Road Management, Valdivia, MOP, 2018.

\begin{tabular}{cc}
\hline Año & $\begin{array}{l}\text { N}^{\circ} \text { de Proyectos en vialidad (obras, } \\
\text { materiales, servicios y suministros) }\end{array}$ \\
\hline 2007 & 37 \\
2008 & 64 \\
2009 & 68 \\
2010 & 76 \\
2011 & 110 \\
2012 & 287 \\
2013 & 119 \\
2014 & 91 \\
2015 & 310 \\
2016 & 100 \\
2017 & 56 \\
\hline
\end{tabular}

Del cuadro anterior se desprenden algunas cuestiones que, tratadas de manera teórica, donde la maquinaria de los actores participantes del poder se legitima a través de estos proyectos que se ejecutan en el espacio urbano. En segundo lugar, que a pesar de las constantes problemáticas que enfrenta proyectos como el de Circunvalación Sur a través del tiempo, primero su paso por el humedal y luego sus inundaciones con consecuencias en viviendas sociales, la tecnocracia propia de la gobernanza neoliberal sigue llevando adelante este tipo de proyectos.

Tercero, que esta alianza público-privada es patente y continua a través del tiempo con consultores que persisten a través de los años en los cuales el proyecto pasa desde su factibilidad hasta su construcción; en cuarto lugar, no sabemos las formas en que se llevaron a cabo las expropiaciones para concretar el proyecto, pero podríamos intuir en que la sustentabilidad $y$ el 
progreso podrían haber actuado como discursos despolitizadores de los grupos o asociaciones colectivas. Por último, hay que señalar que a la par que se lleva a cabo el proyecto de Circunvalación Sur, este promueve el aumento de plusvalías y rentas urbanas, pero sobre todo prepara el terreno para los futuros proyectos inmobiliarios de viviendas sociales que actúan como verdaderos atractores de los excedentes de capital que se producen después de la crisis del 2008, como lo muestra la figura 3.

Tabla 2

Evolución de la construcción de la avenida Circunvalación Sur en Valdivia. Fuente: elaboración propia basado en MOP, 2018.

Table 2

Evolution of the construction of Circunvalación Sur road in Valdivia. Source: own elaboration based on MOP, 2018.

\begin{tabular}{|c|c|c|c|c|}
\hline $\begin{array}{l}\text { Infraestructura } \\
\text { vial }\end{array}$ & Año & Tipo de actividad & $\begin{array}{l}\text { Inversión total } \\
\text { (en M\$ de } \\
\text { millones) }\end{array}$ & Contratista \\
\hline \multirow{9}{*}{$\begin{array}{c}\text { Circunvalación } \\
\text { Sur }\end{array}$} & 2008 & $\begin{array}{l}\text { Estudios de } \\
\text { factibilidad }\end{array}$ & 384.000 & $\begin{array}{l}\text { Axioma } \\
\text { Ingenieros } \\
\text { Consultores } \\
\text { S.A. }\end{array}$ \\
\hline & 2009 & $\begin{array}{l}\text { Estudios de } \\
\text { factibilidad }\end{array}$ & 384.000 & $\begin{array}{l}\text { Luis Valenzuela } \\
\text { Becerra }\end{array}$ \\
\hline & 2010 & $\begin{array}{l}\text { Estudios de } \\
\text { factibilidad }\end{array}$ & 415.790 & $\begin{array}{l}\text { Axioma } \\
\text { Ingenieros } \\
\text { Consultores } \\
\text { S.A. }\end{array}$ \\
\hline & 2011 & $\begin{array}{l}\text { Estudios de } \\
\text { factibilidad }\end{array}$ & 699 & $\begin{array}{l}\text { Empresa de } \\
\text { Ferrocarriles } \\
\text { del Estado }\end{array}$ \\
\hline & 2012 & Expropiación & 6.902 & $\begin{array}{l}\text { Varios } \\
\text { proveedores }\end{array}$ \\
\hline & 2013 & $\begin{array}{l}\text { Asesorías y } \\
\text { consultorías }\end{array}$ & 807.990 & $\begin{array}{l}\text { Jorge Ugarte } \\
\text { Ingenieros } \\
\text { Consultores } \\
\text { E.I.R. L. }\end{array}$ \\
\hline & 2014 & $\begin{array}{l}\text { Asesorías y } \\
\text { consultorías }\end{array}$ & 844.158 & $\begin{array}{l}\text { Jorge Ugarte } \\
\text { Ingenieros } \\
\text { Consultores } \\
\text { E.I.R. L. }\end{array}$ \\
\hline & 2015 & $\begin{array}{l}\text { Asesorías y } \\
\text { consultorías }\end{array}$ & 843.822 & $\begin{array}{l}\text { Jorge Ugarte } \\
\text { Ingenieros } \\
\text { Consultores } \\
\text { E.I.R. L. }\end{array}$ \\
\hline & 2016 & Obras & 18.144 .133 & $\begin{array}{l}\text { Consorcio } \\
\text { Constructor } \\
\text { Puente Santa } \\
\text { Elvira }\end{array}$ \\
\hline
\end{tabular}




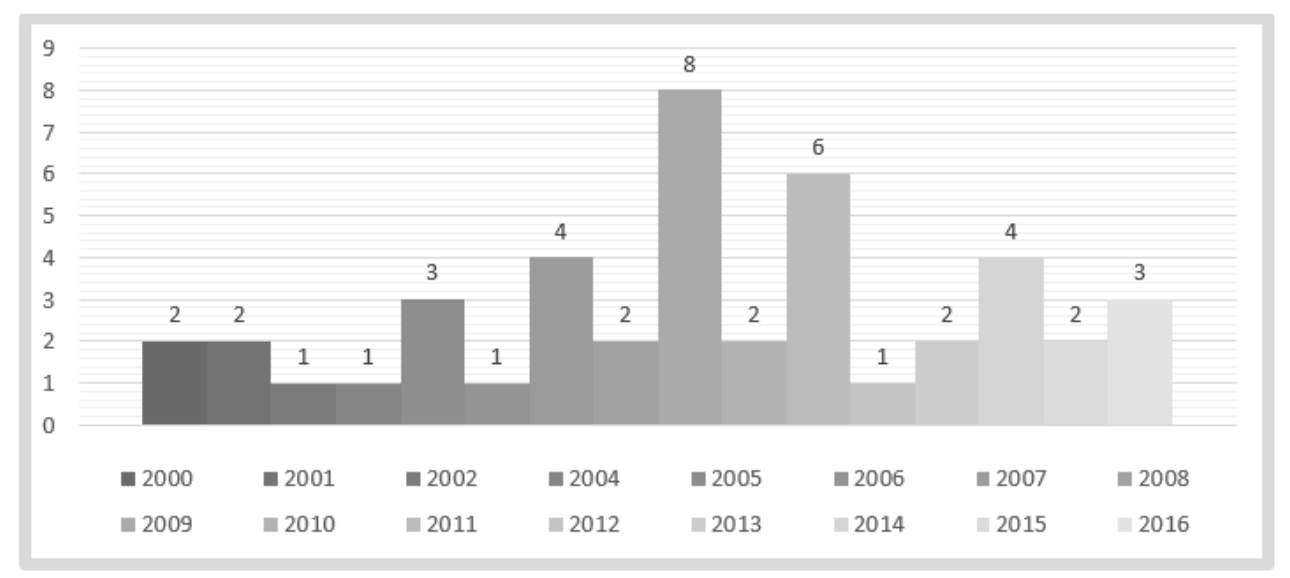

Figura 3. Edificación de viviendas sociales y subsidiadas en Valdivia entre 2000-2016. Fuente: Elaboración propia en base a permisos de edificación Ilustre Municipalidad de Valdivia, 2018.

Figure 3. Construction of social and subsidized housing in Valdivia between 2000-2016. Source:

Own elaboration based on building permits Illustrious Municipality of Valdivia, 2018.

\section{Conclusiones}

La metáfora de la bandurria no es solo un hecho anecdótico dentro de este intrincado proceso de urbanización. Esta representa un hito que hace relucir un block-out energético que hacen reflotar al espacio y el poder en su búsqueda de condiciones de sitio y emplazamiento que puedan no solo ser un contenedor de excedentes de capital, sino que también nichos de renta inmobiliaria y de infraestructura. El contexto espacial (sitio, situación y emplazamiento) relacional es mejor no olvidarlo, como suele suceder.

La despolitización de la naturaleza se legitima desde la alianza de los agentes públicos y privados. Los primeros son un soporte esencial para llevar a cabo los objetivos de los segundos; mientras tanto, aquellos que necesitan invertir sus excedentes esperan que el primero solo les dé el pase. En el intertanto, los actores no autorizados son quienes apoyan las estrategias de la policía para atraer al capital en momento de sobreacumulación.

La preparación de un espacio "natural" como enemigo del progreso es parte de aquella despolitización. Algo que ya había mencionado Marx y que luego sería magistralmente analizado por el geógrafo Neil Smith en la primera mitad de la década de los ochenta. En tal sentido, "la unidad de la naturaleza a la que conduce el capitalismo es ciertamente una unidad materialista pero no es la unidad física o biológica de los científicos naturales. Por el contrario, es una unidad social enfocada en el proceso de producción" (Smith, 2006, p. 46). Esta producción a la que se refiere Smith tiene relación con primera y segunda naturaleza. Siguiendo a Marx, Smith menciona lo siguiente al respecto: "Es imposible alterar cualquier ley natural. Lo que puede modificarse, al cambiar las circunstancias históricas, es la forma en la que operan estas leyes" (Smith, 2006, p. 47). Estos cambios en las circunstancias históricas se espacializan de manera diferenciada. Bajo un contexto neoliberal, la producción del habitar sobre la naturaleza ha llegado a paroxismos notorios. La bandurria como parte de la naturaleza se encontró con la modificación de su espacio: la segunda naturaleza. 


\section{Bibliografía}

Angelo, H. \& Wachsmuth, D. (2015). Urbanizing urban political ecology: a critique of methodological cityism. International journal of urban and regional research, 39(1), 16-27.https://doi. org/10.1111/1468-2427.12105

ATISBA, Estudios \& Proyectos Urbanos. Ministerio de Vivienda y Urbanismo (2005). Asesoría en proyectos urbanos. Guacamayo, Valdivia (Informe final caso cuatro/ Guacamayo, Valdivia). Recuperado de https://docplayer. es/37333372-Asesoria-en-proyectosurbanos.html\#show_full_text

Bolados, P. \& Babidge, S. (2017). Ritualidad y extractivismo. La limpia de canales y las disputas por el agua en el Salar de Atacama-norte de Chile. Estudios atacameños, 54, 201-216. http://dx.doi. org/10.4067/S0718-10432016005000026

Bookchin, M. (1978). Ecology and revolutionary thought. Antipode, 10-11(3-1). https://doi. org/10.1111/j.1467-8330.1978.tb00113.x

Budds, J. (2004). Power, nature and neoliberalism: The political ecology of water in Chile. Singapore journal of tropical geography, 25(3), 322-342. https://doi. org/10.1111/j.0129-7619.2004.00189.x

Budds, J. (2012). La demanda, evaluación y asignación del agua en el contexto de escasez: un análisis del ciclo hidrosocial del valle del río La Ligua, Chile. Revista de geografía norte grande, 52, 167184 . https://doi.org/10.4067/s071834022012000200010

Camacho, F. M. (2012). Competing rationalities in water conflict: Mining and the indigenous community in Chiu Chiu, El Loa Province, northern Chile. Singapore journal of tropical geography, 33(1), 93-107. https://doi.org/10.1111/j.14679493.2012.00451.x

Castree, N. (2007). Making first world political ecology. Environment and planning A, 39(8), 2.030-2.036. https://doi. org/10.1068/a169rev

Castro, E. (2011). Diccionario Foucault. Temas, conceptos y autores. Buenos Aires: Siglo XXI editores. ISBN 978-987-629874-2

Clark, J. (2015). Libertad, igualdad, geografía. Ensayos escogidos de Elisée Reclus. Madrid: Enclave de libros. ISBN 10: 8494270877 . ISBN 13: 9788494270871

Coombes B., Johnson J. T., \& Howitt R. (2012). Indigenous geographies I. Progress in human geography, 36(6), 810-821. https://doi.org/10.1177/0309132511431410

Correia de Andrade, M. (1985). Elisée Reclus. São Paulo: Editora ática.

Delaney, D. (2005). Territory. A short introduction. Oxford: Blackwell publishing. ISBN: 978-0-470-77392-5

Demaria F. \& Schindler S. (2016). Contesting urban metabolism: struggles over waste-to-energy in Delhi, India. Antipode, 48(2), 293-313. https://doi. org/10.1111/anti.12191

Dollfus, O. (1976). El espacio geográfico. Barcelona: Oikos-tau. ISBN 10: 8428103038 ISBN 13: 9788428103039

Emol (7 de febrero de 2017). Ricardo Lagos quiere reactivar el crecimiento económico mediante programa de infraestructura. emol. com. Recuperada de https://www.emol.com/ noticias/Economia/2017/02/07/843855/ Ricardo-Lagos-quiere-reactivar-el- 
crecimiento-economico-medianteprograma-de-infraestructura.html

Engels, F. (2017). Dialéctica de la naturaleza. Madrid: Akal. ISBN 978-84460-4451-2

Espinoza, D. \& Zumelzu Scheel, A. (2016). Valdivia y su evolución post-terremoto 1960: enfoques, factores escalares y condicionantes. Urbano, 19(33), 14-29. Recuperado de http://revistas.ubiobio.cl/ index.php/RU/article/view/2303

Filibi, I. (2005). Democracia más allá del Estado nación: entre la gobernanza y el retorno de lo político. Trabajo presentado en el VII Congreso español de ciencia política y de la administración. Democracia y buen gobierno. Universidad Complutense de Madrid. Madrid.

Harvey, D. (2012). Ciudades rebeldes: Del derecho a la ciudad a la revolución urbana. Madrid: Akal. ISBN 978-84-460-3799-6

Heynen, N., Perkins, H., \& Roy, P. (2006). The political ecology of uneven urban green space. Urban affairs review, 42(1), 3-25. https://doi.org/10.1177/1078087406290729

Hidalgo, R. Rodríguez, L., \& Alvarado, V. (2018). Arriba del cerro o sobre el humedal: producción de naturaleza y expansión inmobiliaria en ciudades marinas y fluviales. El caso de Valparaíso y Valdivia, Chile. Dialogo andino, 56, 87-100. https://doi. org/10.4067/s0719-26812018000200087

Hidalgo, R., Camus, P., Paulsen-Espinoza, A., Olea, J. \& Alvarado, V. (2016). Extractivismo inmobiliario, expoliación de los bienes comunes y esquilmación del medio natural. El borde costero en la macrozona central de Chile en las postrimerías del neoliberalismo. En Innsbrucker Geographische Studien. Band 40 (Eds.). Die Welt verstehen, eine geographische Herausforderung. Eine Festschrift der Geographie Innsbruck für Axel Borsdorf (pp. 251-270). Innsbruck, Austria: Geographie Innsbruck.

Jara, Y. (17 de abril de 2015). Denuncian reiteradas inundaciones en avenida Circunvalación en Valdivia. Radio BioBío Chile. Recuperado de http://www. biobiochile.cl/noticias/2015/04/17/ denuncian-reiteradas-inundaciones-enavenida-circunvalacion-en-valdivia.shtml

Kaika, M. \& Ruggiero, L. (2015). Class meets land: The social mobilization of land as catalyst for urban change. Antipode, 47(3), 708-729. https://doi.org/10.1111/anti.12139

Kay, K. \& Kenney-Lazar, M. (2017). Value in capitalist natures. Dialogues in human geography, 7(3), 295-309. https://doi. org/10.1177/2043820617736584

Lakatos, I. (1998). La metodología de los programas de investigación científica. Madrid: Alianza editorial. ISBN: 9788420623498

Lawhon, M., Ernstson, H. \& Silver, J. (2014). Provincializing urban political ecology: Towards a situated UPE through African urbanism. Antipode, 46(2), 497-516. https:// doi.org/10.1111/anti.12051

Mackinder, H. (1987). El pivote geográfico de la historia. Santiago de Chile: Instituto geopolítico de Chile.

Ministerio de Vivienda y Urbanismo (2016, junio). Ministra Saball constata en terreno la consolidación de conjuntos habitacionales socialmente integrados en Los Ríos. [Sitio web]. Recuperada de http://www.minvu.cl/ opensite_det_20160629181251.aspx?glb

Paulson, S., Gezon L. L. \& Watts, M. (2003). Locating the political in political ecology: 
An introduction. Human organization, 62(3), 205-217. https://doi.org/10.17730/ humo.62.3.e5xcjnd6y $8 v 09 n 6 b$

Postone, M. (2007). Marx reloaded. Repensar la teoría crítica del capitalismo. Madrid: Traficantes de sueños. ISBN: 97884-96453-21-0

Prieto, M. (2015). Bringing water markets down to Chile's Atacama Desert. Water international, 41(2), 191-212. https://doi. org/10.1080/02508060.2015.1107400

Reclus, E. (1875). La Tierra y los hombres. Revista europea, tomo IV(70), 663-664. Recuperado de http://www.filosofia.org/ rev/reu/1875/n070p663.htm

Raffestin, C. (2015). Por una geografía del poder. Michoacán: El colegio de Michoacán. ISBN: 978-607-8257-41-6

Rancière, J. (2006). Diez tesis sobre la política. En J. Rancière, Política, policía, democracia (pp. 59-79). Santiago: Lom.

Rojas, C., Sepúlveda-Zúñiga, E., Barbosa, O., Rojas, O. \& Martínez, C. (2015). Patrones de urbanización en la biodiversidad de humedales urbanos en Concepción metropolitano. Revista de geografia norte grande, 61, 181$204 . \quad$ https://doi.org/10.4067/s071834022015000200010

Romero, H., Videla, A., \& Gutiérrez, F. (2017). Explorando conflictos entre comunidades indígenas y la industria minera en chile: las transformaciones socioambientales de la región de Tarapacá y el caso de Lagunillas. Estudios atacameños, 55, 231-250. http://dx.doi. org/10.4067/S0718-10432017005000019

Roth, R. J. \& Dressler, W. (2012). Marketoriented conservation governance: The particularities of place. Geoforum, 43(3), 363-366. https://doi.org/10.1016/j. geoforum.2012.01.006

Santos, M. (2000). La naturaleza del espacio. Técnica y tiempo. Razón y emoción. Barcelona: Ariel. ISBN: 9788434434608

Schorr, B. (2018). Oportunidades desiguales: empresas y Estado en conflictos sobre la minería en Chile. Estudios atacameños, 57, 239-255. http://dx.doi. org/10.4067/S0718-10432018005000601

Signorelli, A. (1999). Antropología urbana. Madrid: Anthropos. ISBN 847658-562-4

Silva, P. (2010). En el nombre de la razón. Tecnócratas y política en Chile. Santiago de Chile: Ediciones Universidad Diego Portales. ISBN 9563141172, 9789563141177

Smith, N. (2006). La producción de la naturaleza. La producción del espacio. Ciudad de México: UNAM. ISBN: 970323741X, 9789703237418

Sultana, F. (2011). Suffering for water, suffering from water: Emotional geographies of resource access, control and conflict. Geoforum, 42(2), 163-172. https:// doi.org/10.1016/j.geoforum.2010.12.002

Spinoza, B. (2011). Ética demostrada según el orden geométrico. Madrid: Alianza editorial. (Trabajo original publicado en 1675).

Swyngedouw, E. \& Heynen, N. (2003). Urban political ecology, justice and the politics of scale. Antipode, 35(5), 898918. https://doi.org/10.1111/j.14678330.2003.00364.x 
Swyngedouw, E. (2011a). Interrogating post-democratization:

Reclaiming

egalitarian political spaces. Political geography, 30(7), 370-380. https://doi. org/10.1016/j.polgeo.2011.08.001

Swyngedouw, E. (2011b). ¡La naturaleza no existe! La sostenibilidad como síntoma de una planificación despolitizada. Urban, 1, 41-66. Recuperado de http://polired.upm. es/index.php/urban/article/view/410

Swyngedouw, E. (2014). Where is the political? Insurgent mobilisations and the incipient "return of the political". Space and polity, 18(2), 122-136. https://doi.org/ 10.1080/13562576.2013.879774

Turner, M.D. (2017). Political ecology III. Progress in human geography, 41(6), 795-802. https://doi. org/10.1177/0309132516664433
Vidal de la Blache, P. (1902). Les conditions géographiques des faits sociaux. Annales de géographie, 11(55), 13-23. https://doi. org/10.3406/geo.1902.18145

Walker, P. (2005). Political ecology: where is the ecology? Progress in human geography, 29(1), 73-82. https://doi. org/10.1191/0309132505ph530pr

Wilson, J. \& Bayón, M. (2017). Fantastical materializations: Interoceanic infrastructures in the Ecuadorian Amazon. Environment and planning D: Scociety and space, 35(5), 836-854. https://doi. org/10.1177/0263775817695102

Zunino, H. (2006). Power relations in urban decision-making: neo-liberalism, "techno-politicians" and authoritarian redevelopment in Santiago, Chile. Urban studies, 43(10), 1.825-1.846. https://doi. org/10.1080/00420980600838184 